\title{
LOCAL FUNCTIONALS AND GENERALIZED RANDOM FIELDS ${ }^{1}$
}

BY M. M. RAO

\author{
Communicated by Henry McKean, October 13, 1967
}

1. Introduction. Let $\mathfrak{K}\left(R^{n}\right)$ be the Schwartz space of infinitely differentiable real functions on $R^{n}$, the Euclidean $n$-space, with compact supports, and let $F: \mathfrak{K}\left(R^{n}\right) \rightarrow \widetilde{R}$ be a map where $\widetilde{R}$ is the class of real random variables on a (fixed) probability space. Then $F$ is said to be a generalized random field (process if $n=1$ ) if it is linear and continuous. Here continuity means that if $\left\{f_{m}\right\} \subset \mathcal{K}\left(R^{n}\right)$ and $f_{m} \rightarrow 0$ in the topology of $\mathscr{K}\left(R^{n}\right)$, then $F\left(f_{m}\right) \rightarrow 0$ in probability. Such an $F$ is said to have independent values if $F(f)$ and $F(g)$ are mutually independent, whenever $f, g$ in $\Re\left(R^{n}\right)$ have disjoint supports. Let $M(\cdot): \Re\left(R^{n}\right)$ $\rightarrow$ scalars, be a functional such that (i) $M(\cdot)$ is bounded on bounded sets of $\Re\left(R^{n}\right)$; (ii) $M(\cdot)$ is continuous in that for any $\epsilon>0$, there is a neighborhood $V$ of zero in $\Re\left(R^{n}\right)$ such that $f-g \in V$ implies $|M(f)-M(g)|<\epsilon$; and (iii) $M(f+g)=M(f)+M(g)$ whenever $f$ and $g$ have disjoint supports. Such a functional $M(\cdot)$ is termed local by Gel'fand and Vilenkin [4, Chapter III, \$4.1, footnote 2] where they raised the problem of characterizing $M(\cdot)$. Local functionals play a key role in the theory of generalized random fields with independent values. A special form of $M(\cdot)$ was used by Gel'fand in his study of such generalized random processes (cf. [3] and [4]). The purpose of this note is to state some results on the characterizations of local functionals and, as applications, to present generalizations of the Lévy-Khintchine representation formulas for characteristic functionals of generalized random fields with independent values. These results extend and complete the fundamental work of Gel'fand (cf. [3] and [4]) in many ways. The proofs and subsidiary results will be given elsewhere.

The concept of generalized random processes was independently introduced by Gel'fand [3] and Itô [5] who studied the generalized processes with independent values and the generalized stationary processes, respectively (see also [10]). The latter results were extended to certain nonstationary random fields in [7]. Another aspect of the theory was considered by Urbanik [9], for processes with independent values.

\footnotetext{
1 This research was supported under the NSF grants GP-5921 and GP-7678.
} 
2. Local functionals. Following [4], a local functional $M(\cdot)$ on $\mathscr{K}\left(R^{n}\right)$ is said to be of order $m(<\infty)$ if $M(\cdot)$ is continuous in the topology of $\varkappa^{m}\left(R^{n}\right)$, the space of $m$ times, continuously differentiable real functions with compact supports, on $R^{n}$. (For the theory of these spaces, see [8].) Also a subset $N$ of $R^{n}$ is said to be the support of $M(\cdot)$ if: (a) $N$ is closed, (b) for every open set $V$ in $R^{n}$ such that $V \cap N \neq 0$; there is a $g$ in $\Re\left(R^{n}\right)$ with support in $V \cap N$ such that $M(g) \neq 0$; and (c) for every $f$ in $\mathscr{K}\left(R^{n}\right)$ with support in $R^{n}-N$, $M(f)=0$. Now the following characterizations hold.

Theorem 1. If $M(\cdot)$ is a local functional on $\mathfrak{K}\left(R^{n}\right)$ of order $m \geqq 0$, then it can be represented as $\left(D=\partial /\left(\partial t_{1} \cdots \partial t_{n}\right)\right.$, etc)

(1) $M(f)=\int_{R^{n}} \Phi\left(f(t), D f(t), \cdots, D^{m} f(t), t\right) d \mu(t), \quad f \in \mathfrak{K}\left(R^{n}\right)$,

where $\mu$ is a Borel measure in $R^{n}$ which is bounded on compact sets and where (i) $\Phi(\cdot, \cdot, \cdots, t)$ is continuous for almost all $t$; (ii) $\Phi(0,0, \cdots, 0, t)=0$, a.a. $(t)$; (iii) $\Phi(x, y, \cdots, z, \cdot)$ is a Borel function for each real $x, y, \cdots, z$; and (iv) for each compact $A \subset R^{n}$ and each $\delta>0$ such that $f \in \mathscr{K}\left(R^{n}\right), \operatorname{supp}(f) \subset A$ and $\left|\left(D^{i f}\right)(x)\right| \leqq \delta, 0 \leqq i \leqq m$, implies $\left|\Phi\left(f(t), D f(t), \cdots, D^{m} f(t), t\right)\right| \leqq c(A, \delta)<\infty$ a.a. $(t)$, where $c(A, \delta)$ is a constant depending only on $A$ and $\delta$. In particular, if $M(\cdot)$ has compact support then it is of finite order and a representation of type (1) holds for some $m \geqq 0$. Conversely, a function $\Phi$ and a Borel measure $\mu$ satisfying the above conditions define a local functional on $\nVdash\left(R^{n}\right)$ through (1).

If $\tau_{h}$ is the translation operator on $\Re\left(R^{n}\right)$, so that $\tau_{h} f(x)=f(x+h)$, a local functional $M(\cdot)$ is translation invariant if $M\left(\tau_{h} f\right)=M(f)$, $f \in \mathcal{K}\left(R^{n}\right), h \in R^{n}$. For such functionals the above result can be refined as follows.

THEOREM 2. If $M(\cdot)$ is a translation invariant local functional on $\mathfrak{K}\left(R^{n}\right)$ of order $m \geqq 0$, then it is given by

$$
M(f)=\int_{R^{n}} \Phi\left(f(t), D f(t), \cdots, D^{m} f(t)\right) d \mu(t), \quad f \in \mathfrak{K}\left(R^{n}\right),
$$

where $\Phi(0, \cdots, 0)=0, \Phi$ is a continuous function satisfying conditions (i)-(iv) of Theorem 1, and $\mu$ is the Lebesgue measure. Similar statements hold for $M(\cdot)$ with compact support.

The next result generalizes the above in a different direction. 
Theorem 3. Let $G$ be a locally compact topological group and $C_{\infty}(G)$ be the space of real continuous functions on $G$ with compact support. If $M(\cdot)$ on $C_{\infty}(G)$ is a local functional, then it can be represented as

$$
M(f)=\int_{G} \Psi(f(g), g) d \mu(g), \quad f \in C_{\infty}(G),
$$

where $\mu$ is a Borel measure which is bounded on compact sets and where $\Psi$ on $R \times G$ satisfies; (i) $\Psi(0, g)=0$, a.a. $(g)$; (ii) $\Psi(\cdot, g)$ is continuous, a.a. $(g)$; (iii) $\Psi(x, \cdot)$ is a Borel function for $x \in R$; and (iv) for each compact $\mathrm{A} \subset G$ and each $\delta>0$ such that $f \in C_{\infty}(G)$, supp $(f) \subset A$ and $|f| \leqq \delta$ implies $|\Psi(f(g), g)| \leqq c_{1}(A, \delta)<\infty$, a.a. $(g)$, where the constant $c_{1}(A, \delta)$ depends only on $A$ and $\delta$. If moreover $M(\cdot)$ is translation invariant on $C_{\infty}(G)$, then $\Psi$ on $R \times G$ of (3) can be chosen to be a function of the first variable only (so that $\Psi$ is defined on $R$ and satisfies (i)-(iv)) and $\mu$ is the Haar measure on $G$. If $M(\cdot)$ has compact support (but not necessarily translation invariant), then the measure $\mu$ of (3) above is also bounded.

REMARK. A special case of Theorem 3, when $C_{\infty}(G)$ is replaced by the space of bounded functions on $G$ vanishing off compact sets and $G$ is abelian, was obtained by Allan D. Martin [6]. (Unfortunately Martin died in September 1964, and his results were unpublished.)

3. Generalized random fields. If $F: \Re\left(R^{n}\right) \rightarrow \widetilde{R}$ is a generalized random field, its characteristic functional $L_{0}(\cdot)$ is defined by $L_{0}(f)=$ $E\left(e^{i F(f)}\right)$ where $E$ is the expectation operator. If $F$ has independent values, then $L_{0}\left(f_{1}+f_{2}\right)=L_{0}\left(f_{1}\right) \cdot L_{0}\left(f_{2}\right)$ for $f_{1}, f_{2}$ in $\mathcal{K}\left(R^{n}\right)$ of disjoint supports and conversely (cf. [4]). Now the following result, on the structure of certain functionals can be given. First define a functional $L(\cdot)$ as

$$
L(f)=e^{M(f)}, \quad f \in \mathscr{K}\left(R^{n}\right) .
$$

Theorem 4. Suppose a functional $L(\cdot)$ on $\mathscr{K}\left(R^{n}\right)$ is given by (4) where $M(\cdot)$ is an arbitrary local functional of finite order, say $m$, on $\Re\left(R^{n}\right)$. Then $L(\cdot)$ is a characteristic functional (necessarily of some generalized random field with independent values) if and only if

$$
\exp \left(\int_{A} \Phi\left(x_{1}, \cdots, x_{\nu}, t\right) d \mu(t)\right), \quad x_{i} \in R
$$

is positive definite as a function of $x_{1}, \cdots, x_{\nu}$ for all compact $A \subset R^{n}$, where $\Phi$ and $\mu$ are the same functions associated with $M(\cdot)$ through (1), the $\nu$ being the total number of partial derivatives of order $\leqq m$. 
The necessity of (5) above answers affirmatively a question in $[4$, p. 285], even without translation invariance. The next result, which generalizes and completes the theory of Gel'fand [3], [4] in many ways, is a considerable extension of the Lévy-Khintchine representation formula for the characteristic functions of stochastic processes without fixed points of discontinuity and with independent (but not necessarily stationary) increments (cf. [2, p. 421, Equation (7.5)]) to the generalized random fields with independent (but not necessarily stationary) values. [A generalized random field has (strictly) stationary values if its characteristic functional $L_{0}(\cdot)$ satisfies $L_{0}\left(\tau_{h} f\right)=L_{0}(f), f \in \mathfrak{K}\left(R^{n}\right), h \in R^{n}$.]

THEOREM 5. Let $L(\cdot)$ on $K\left(R^{n}\right)$ be any functional given by (4) where $M(\cdot)$ is a local functional of order $m$ on $\Re\left(R^{n}\right)$. Suppose the measure $\mu$ associated with $M(\cdot)$ by (1) is nonatomic. Then $L(\cdot)$ is a characteristic functional of a generalized random field on $\nVdash\left(R^{n}\right)$ with independent values if and only if it can be given as

$$
L(f)=\exp \left\{\int_{R^{n}} \Phi\left(f(t), D f(t), \cdots, D^{m} f(t), t\right) d \mu(t)\right\}, \quad f \in \mathcal{K}\left(R^{n}\right),
$$

where $\Phi$ is given by the following formula.

$$
\begin{aligned}
\Phi(\mathbf{x}, t)= & \int_{|\mathbf{y}|>0}\left[e^{i(\mathbf{x}, y)}-\alpha(y)(1+i(\mathbf{x}, y))\right] \sigma(d y ; t) \\
& +a_{0}(t)+\sum_{|k|=1}^{2} a_{k}(t) \frac{(i \mathrm{x})^{k}}{k !},
\end{aligned}
$$

where $\sigma(\cdot, t)$ is a positive tempered measure in $R^{v}$ for each $t \in R^{n},(x, y)$ is the inner product in $R^{v}, \sigma(A, \cdot)$ is a Borel function for each Borel set $A$ of $R^{\nu}$, and where for almost all $t \in R^{n}$ the following conditions hold:

$$
\begin{aligned}
& \int_{0<|y| \leqq 1}|y|^{2} \sigma(d y ; t)+\int_{|y|>1} \sigma(d y ; t)<\infty, \\
& \int_{|y|>0}(1-\alpha(y)) \sigma(d y ; t)+a_{0}(t)=0, \quad \sum_{|r|=|\varepsilon|=1} a_{r+8}(t) \xi_{r} \bar{\xi}_{s} \geqq 0 .
\end{aligned}
$$

Here the $a(\cdot)$ 's are some Borel functions on $R^{n}$ depending on $\Phi$ and $\alpha$ where $\alpha(\cdot)$ is an entire analytic function of exponential type (i.e. $\alpha \in Z$ in the terminology of $[4$, Chapter I]) such that $\alpha(y)-1$ has a zero of order 3 at $y=0$. (Also $|k|=k_{1}+\cdots+k_{\nu},|y|^{2}=y_{1}^{2}+\cdots+y_{\nu}^{2}$, $\xi^{\prime}$ s are complex numbers, $k !=k_{1} ! \cdots k_{\nu} !$, and $x^{k}=x_{1}^{k_{1}} \cdots x_{\nu}^{k_{\nu}}$, as in [8].) 
Finally, if $L(\cdot)$ is also translation invariant, then $\Phi$ of (7) does not involve the last variable t and then $\sigma$ and $a$ also do not involve $t$. (See [4] for the concept of tempered measure and the properties of the space $Z$.)

4. Remarks. (a) Choosing $\alpha(\cdot)$ appropriately in (7), one can deduce the classical Lévy-Khintchine formula [2, p. 421]. Also an exactly similar result holds for generalized random processes, with independent values, on $C_{\infty}(G)$, using Theorem 3.

(b) A representation of a general local functional $M(\cdot)$ as $M(\cdot)$ $=\sum_{n} M_{n}(\cdot)$ where $M_{n}$ is of finite order is not necessarily true, in contrast to the situation for distributions (cf. [8, p. 95, Théorème $29]$ ). In this sense Theorem 1 is the most general result obtainable on representations of $M(\cdot)$ and similarly Theorem 5 is a very general result in this direction. [However, the sufficiency of the latter result holds without the condition of nonatomicity of $\mu$.]

(c) The proofs of the results of $\$ 2$ employ and extend, in a detailed analysis, the results and techniques of [1]. Then the proofs of the results of $\$ 3$ use this representation theory and several results of Chapters II and III of [4]. To prove the necessity of (5) one injects the space $\varkappa^{m}\left(R^{n}\right)$ in a certain subspace of $\Gamma^{\nu}\left(R^{n}\right)$ suitably, where $\Gamma^{v}$ is the space of vector valued (with $\nu$-components) continuous functions on $R^{n}$ with compact supports, and then associates a continuous functional $\bar{L}(\cdot)$ on $\Gamma^{\nu}$ whose restriction on the subspace is in one-toone correspondence with $L(\cdot)$ of $(4)$. The result is then deduced, by showing that $\bar{L}(\cdot)$ is positive definite. The sufficiency of (5) and the proof of Theorem 5 are obtained by extending the methods of [4]. The formula (6) is useful for further study of the generalized random fields with independent values, in the same way as the classical formula is useful in the study of processes with independent increments.

(d) (Added in proof.) Concerning Theorem 1, one can make the following apparently more general statement. By considering $c^{\infty}$ partitions of unity in $\nVdash$ subordinate to a given countable covering $\left\{A_{i}\right\}$ of $R^{n}$ which (by refinement if necessary) may be assumed locally finite and $A_{i}$ relatively compact, one has $M(f)=\lim _{r \rightarrow \infty} M_{r}(f), f \in \Re$, where $M_{r}(\cdot)$ is a local functional on $\mathscr{K}$ of compact support, contained in $A_{r}$, so that it has finite order $m_{r}$. This yields the most general form of the formula (1) as

$$
M(f)=\lim _{r \rightarrow \infty} \int_{R^{n}} \Phi_{r}\left(f(t), D f(t), \cdots, D^{m_{r}} f(t), t\right) d \mu_{r}(t), \quad f \in \mathcal{K},
$$

where $\left\{m_{r}\right\}$ is a (not-necessarily bounded) set of positive integers, and $\left\{\Phi_{r}\right\},\left\{\mu_{r}\right\}$ satisfy the corresponding conditions of Theorem 1 
(given for $\Phi, \mu$ ). Similar statements hold with regard to Theorems 2 , 4 and 5.

I thank Henry McKean for helpful comments and questions on the material of this paper.

\section{REFERENCES}

1. R. V. Chacón and N. Friedman, Additive functionals, Arch. Rat. Mech. Anal. 18 (1965), 230-240.

2. J. L. Doob, Stochastic processes, Wiley, New York, 1953.

3. I. M. Gel'fand, Generalized random processes, Dokl. Acad. Nauk. SSSR 100 (1955), 853-856 (Russian).

4. I. M. Gel'fand and N. Ya. Vilenkin, Generalized functions. Vol. 4, Moscow, 1961; English transl., Academic Press, New York, 1964.

5. K. Itô, Stationary random distributions, Mem. Colloq. Sci. Univ. Kyoto, Ser. A 28 (1954), 209-223.

6. A. D. Martin, Oral communication, 1963.

7. M. M. Rao, Characterization and extension of generalized harmonizable random fields, Proc. Nat. Acad. Sci. U.S.A. 58 (1967), 1213-1219.

8. L. Schwartz, Théorie des distributions. Vol. I, Actualites Sci. Indust., no. 1091, Hermann, Paris, 1950.

9. K. Urbanik, Generalized stochastic processes with independent values, Proc. Fourth Berkeley Sympos. Math. Statist. and Prob. 2 (1961), 569-580.

10. A. M. Yaglom, Some classes of random fields in n-dimensional space related to stationary random processes, Theor. Veroyatnost. i Primenon. 2 (1957), 292-338.

Carnegie-Mellon University 